\title{
NEW YORK ACADEMY OF MEDICINE
}

A Symposium on the Present Status of Cortisone and ACTH was presented on January 12, 1950, at a special meeting arranged by the Committee on Medical Education under the chairmanship of Dr. R. F. Loeb. The seven papers abstracted below were published in full in the Bulletin of the New York Academy of Medicine (1950), 2nd Series, Vol. 26, No. 4.

Effects of Cortisone and ACTH in Rheumatoid Arthritis. By R. H. Freyberg.

The author reviews the effects of treating 169 cases of chronic rheumatic disease with cortisone and 175 with ACTH (the total so far treated in America). Cortisone and ACTH are effective in the treatment of rheumatoid arthritis, rheumatoid spondylitis, Still's disease, and psoriasis with arthritis. The average dosage for cortisone is $100 \mathrm{mg}$. daily, in one injection, until a remission is induced, and then $100 \mathrm{mg}$. thrice weekly. With ACTH it seems preferable to give small doses (generally $25 \mathrm{mg}$. of standard preparation) four times daily. Relapse after cessation of treatment usually occurs rapidly. Complications include: oedema from sodium retention, hypopotassiumaemia, partial hypercortico-adrenalism, menstrual disorders, diabetic carbohydrate metabolism, insomnia, tachycardia, excitability, and mental depression. They occur more frequently in women, particularly those of premenopausal age, and in children, who require comparatively larger doses.

Substances chemically similar to cortisone studied to date, include compound S, progesterone, 17-hydroxyprogesterone, pregnenolone, acetoxy-pregnenolone and testosterone, but all are ineffective. In conclusion it is emphasized that, since prolonged administration of cortisone may lead to adrenal atrophy and that of ACTH to pituitary atrophy with adrenal hyperplasia, further research on this problem is required before the hormones can be considered for practical therapy. It remains, however, the greatest advance in the treatment of rheumatic diseases.

The Effect of Cortisone and ACTH on Rheumatic Fever. By C. McEwen, J. J. Bunim, J. S. Baldwin, A. G. Kuttner, S. B. Appel, and A. J. Kaltman.

The results of cortisone and ACTH therapy in three children with acute rheumatic fever are described, and the authors also summarize the current literature on the subject. It is confirmed that fever, polyarthritis, "rheumatic toxicity", and nodules, responded rapidly to treatment with either cortisone or ACTH. The sedimentation rate, leucocyte count, and haemoglobin concentration, returned more slowly towards normal, and a slight rise in serum albumin with a fall in the globulin was noted. Pericardial friction rubs and atrioventricular conduction defects cleared rapidly but may improve spontaneously. Complications included salt- and water-retention, transient hypertension, glycosuria, mental disturbances, hypocalcaemia, a peculiar facial eruption, and some features of Cushing's syndrome. The effect of the therapy on the carditis was more difficult to assess and the authors conclude that further study of a larger number of cases with carditis is necessary before the value of cortisone and ACTH in this condition can be assessed.

\section{The Treatment of Disseminated Lupus Erythematosus with Cortisone and Adrenocortico-} tropin. By G. Baehr and L. J. Soffer.

The results of treating five cases of disseminated lupus erythematosus (four women, and one boy of 13 years) are discussed. It was found that the optimum dosage was 150$200 \mathrm{mg}$. cortisone, or $100 \mathrm{mg}$. ACTH, given daily in four divided doses. When the rash 
and other signs of the disease had disappeared the dose was reduced to 50-100 mg. cortisone, or $75-100 \mathrm{mg}$. ACTH. In order to stimulate adrenal cortical activity cortisone was replaced by ACTH as soon as the maintenance dose of cortisone had been determined. The amount of ACTH was then gradually reduced over 4 to 6 weeks until the patients' own adrenals assumed the full load.

Complications included sodium retention, hypertension, hypopotassiumaemia, and cerebral disturbances. Details of treatment of these conditions are given. Improvement was dramatic in all cases, but it is stressed that the therapy must be considered as producing a remission and not as a curative procedure, since leucopenia, raised sedimentation rate, and the presence of "L.E." cells in the bone marrow, persisted after apparent clinical recovery.

The Use of ACTH and Cortisone in Neoplastic Disease. By O. H. Pearson, L. P. Eliel, and T. R. Talbot, Jr.

Five cases of acute leukaemia (one lymphocytic and four granulocytic) were treated with $\mathrm{ACTH}$, in doses of $50 \mathrm{mg}$. daily for children and $100 \mathrm{mg}$. daily for adults. In all cases there was a dramatic improvement, with rapid shrinkage of spleen, liver, and lymph nodes, and some improvement in the blood picture as shown by increased maturity of circulating leucocytes and marked reticulocytosis. Similar improvement was seen in the bone marrow, but in no case did the blood or bone marrow return to normal, and in one case a relapse occurred three weeks after discontinuing ACTH.

Two cases of acute leukaemia which had previously received antifolic treatment died after beginning ACTH therapy, but both were already critically ill. One case each of carcinoma of the breast, embryonal carcinoma of the testis or adrenal, Ewing's tumour, plasma cell myoma, rhabdomyosarcoma, and carcinoma of the pancreas, showed no definite response to ACTH.

The authors have little experience of the use of cortisone in acute leukaemia, but one child showed a good remission whereas another died 48 hours after starting cortisone therapy. It is concluded that ACTH can produce remissions in cases of acute leukaemia but is not curative.

\section{Physiology of the Pituitary-Adrenal System. By Jane A. Russell.}

In reviewing the chemical composition of the known adrenal steroids, the author points out that the six or seven hormones which are active in maintaining life in adrenalectomized animals may be divided into those oxygenated at the 11-carbon atom and those not so oxygenated (desoxy type). Cortisone (compound B) and 11-dehydrocorticosterone (compound $\mathrm{A}$ ) are less active than 17- $\mathrm{OH}, 11$-dehydrocorticosterone (compound $\mathrm{E}$ or cortisone); the most potent, but the most difficult to synthesize, is $17-\mathrm{OH}$ corticosterone (compound F).

The physiological effects of these hormones are described as follows:

(1) Maintenance of salt and water balance is demonstrable since lack of the hormones gives rise to salt depletion, dehydration, and rise in the plasma potassium, whereas overdosage, as in treatment with cortisone, may lead to salt-retention and oedema.

(2) Lack of corticosterones gives rise to increased nitrogen loss and depletion of muscle glycogen, the site of action probably being on some phase of gluconeogenesis from body protein. Excess hormone therapy has also a diabetogenic effect, the desoxy compounds being less active in this respect.

(3) Involution in lymphoid tissue and decrease in circulating eosinophils occur in hormone deficiency and the hormones also appear to affect the spreading factor in the skin, probably by inhibition of hyaluronidase.

(4) Resistance to stress and adaptation to environmental change is diminished by hormone lack, particularly by lack of the 11-oxygenated steroids, although the mode of action is unknown in this respect. 
Experimentally ACTH has the same effect as the corticosteroids, but functioning adrenal tissue is necessary for its effective action. It has also been shown that the adrenals are practically inactive in the absence of pituitary secretion.

The Effect of ACTH and Cortisone on Connective Tissue. By C. Ragan, E. L. Howes, C. M. Plotz, K. Meyer, J. W. Blunt, and R. Lattes.

The authors give a brief account of experiments to show the effect of cortisone and ACTH on wound healing. Skin defects were made in the ears of one series of rabbits by the Howes method, and femoral fractures were made in a second series by the Hudack and Blunt technique.

It was found that there was marked delay in wound healing and fibroblast reaction in the animals previously treated with ACTH or cortisone compared with the controls. Large doses of cortisone, equivalent to $500 \mathrm{mg}$. daily in a $50-\mathrm{kg}$. adult, seemed completely to suppress granulation-tissue formation, whereas smaller doses merely delayed its appearance.

It is suggested that the hyperadrenal state renders the mesenchymal tissues incapable of reacting to trauma from whatever cause, and that this may be the reason for a similarity in remission of symptoms in clinically different diseases treated with ACTH or cortisone.

The Relationship of Adrenal Cortical Activity to Immune Responses. By E. E. Fischel.

A series of experiments is described to show the effect of ACTH and cortisone on the development of immune or allergic reactions, and the findings of other workers on similar experiments are discussed.

Using Kabat's quantitative technique for the induction of anaphylaxis, it was found that the response of guinea-pigs was unaffected by treatment with ACTH. The Arthus reaction was used for a quantitative study in rabbits and guinea-pigs, but there was no appreciable difference in reaction between controls and ACTH-treated animals. The anamnaestic response was studied in rabbits using crystalline egg albumin, but no rise occurred in the antibody content of the serum of animals treated with ACTH. Finally, hyperglobulinaemia was produced in rabbits by a pneumococcal immunization technique, and it was apparent that antibody formation was significantly diminished in ACTHtreated animals.

It is concluded that ACTH and cortisone do not alter the union of antigen and antibody but may have some effect on the formation of antibody. Further study is recommended. 